\title{
Chess knowledge predicts chess memory even after controlling for chess experience: Evidence for the role of high-level processes
}

\author{
David M. Lane ${ }^{1,2}$ - Yu-Hsuan A. Chang ${ }^{1}$
}

Published online: 3 November 2017

(C) Psychonomic Society, Inc. 2017

\begin{abstract}
The expertise effect in memory for chess positions is one of the most robust effects in cognitive psychology. One explanation of this effect is that chess recall is based on the recognition of familiar patterns and that experts have learned more and larger patterns. Template theory and its instantiation as a computational model are based on this explanation. An alternative explanation is that the expertise effect is due, in part, to stronger players having better and more conceptual knowledge, with this knowledge facilitating memory performance. Our literature review supports the latter view. In our experiment, a sample of 79 chess players were given a test of memory for chess positions, a test of declarative chess knowledge, a test of fluid intelligence, and a questionnaire concerning the amount of time they had played nontournament chess and the amount of time they had studied chess. We determined the numbers of tournament games the players had played from chess databases. Chess knowledge correlated .67 with chess memory and accounted for $16 \%$ of the variance after controlling for chess experience. Fluid intelligence accounted for an additional 13\% of the variance. These results support the conclusion that both high-level conceptual processing and low-level recognition of familiar patterns play important roles in memory for chess positions.
\end{abstract}

Keywords Chess $\cdot$ Memory $\cdot$ Expertise $\cdot$ Knowledge $\cdot$ CHREST

David M. Lane

lane@rice.edu

1 Rice University, Houston, TX, USA

2 Department of Psychology, MS-25, Rice University, 6100 Main Street, Houston, TX 77054, USA
The strong relationship between chess expertise and memory for chess positions is one of the most robust findings in cognitive psychology (Chase \& Simon, 1973; Charness, 1981; de Groot, 1965; Gobet \& Clarkson, 2004; Gobet \& Simon, 1996; Goldin, 1978; Gong, Ericsson, \& Moxley, 2015; Lane \& Robertson, 1979). However, despite over half a century of research, the basis of this relationship is not yet settled. Chase and Simon (1973) theorized that chunks based on configurations of pieces learned while playing and studying chess are stored in long-term memory. These chunks are recognized during the presentation of a chess position, and labels for the chunks are stored in short-term memory for later recall. The key finding regarding the effect of expertise was that experts recalled larger chunks, enabling them to recall more pieces. According to this view, as players have more experience with chess positions, they are able to recognize more and larger patterns of pieces, thus allowing more accurate recall (Gobet, 1998a, 2013; Gobet \& Simon, 2000; Schultetus \& Charness, 1999).

Research has contradicted the notion that memory for chess positions can be explained in terms of limits on the number of chunks that can be held in short-term memory. One of the hallmarks of short-term memory is that it is easily disrupted by the performance of an interpolated task. This is not the case, however, for memory for chess positions (Charness, 1976). Moreover, the finding that experts can remember two chess positions almost as well as they can recall one (Cooke, Atlas, Lane, \& Berger, 1993; Frey \& Adesman, 1976; Gobet \& Simon, 1996) is also inconsistent with the notion that recall is based on short-term memory.

Gobet and Simon (1996) extended the original Chase and Simon (1973) chunking theory to account for these findings. According to their extension, which they named template theory, chess players learn large chunks of pieces, called "templates," from exposure to thousands of chess games. Such templates differ from standard chunks not only in that they are 
larger, but also in that they contain "slots" for as-yet-unspecified pieces. Template theory is instantiated by a computational model called CHREST (Gobet \& Simon, 2000). In CHREST, the learning and recall of chunks and templates involves the relatively low-level cognitive process of recognizing patterns of pieces (chunks) and patterns of chunks (templates).

An important aspect of chunking theories of chess memory (Gobet, 1996; Simon \& Gilmartin, 1973) is that they model the learning of chunks and, in the case of CHREST, the learning of templates. Learning is based on the elementary perceiver and memorizer (EPAM) model (Feigenbaum, 1961; Feigenbaum \& Simon, 1984; Richman, Staszewski, \& Simon, 1995). In EPAM models, learning occurs automatically with the presentation of stimuli. Reoccurring patterns of stimuli are learned, and these patterns provide the basis of recall. No high-level concepts are hypothesized to be involved in the learning of these patterns.

CHREST has been very successful in accounting for many key findings in chess memory, including the large expertise effect for realistic chess positions, the much better recall of realistic than of random positions, and the small expertise effect for random positions. Moreover, CHREST has been said to account for chess memory findings better than other theories and to be "sufficient to reproduce the data under study." (Gobet, 2016, pp. 57-58). A strength of CHREST is that, as a computational model, it is able to produce the behavior it is modeling. Because there can be no ambiguity about its predictions, CHREST has been cited as an example of why computational models are preferable to "verbal theories" (Gobet, Lane, \& Lloyd-Kelly, 2015).

CHREST's success shows convincingly that pattern recognition is an important component of memory for chess positions. Although CHREST could, in principle, also include high-level representations, such as the opening from which a position was derived and potential plans and moves in the position (Gobet \& Simon, 1996), CHREST currently implements little of this kind of information. Referring to high-level conceptual processes, Gobet and Simon (1996) stated explicitly that their explanation "does not require these concepts" ( $p$. 19). CHREST (Gobet, 2016; Gobet et al., 2001) continues to model the learning of templates as being performed automatically via pattern recognition.

Research conducted outside the domain of chess has provided considerable evidence that conceptual processing plays an important role in memory (Baddeley \& Hitch. 2017; Bartlett, 1932; Bransford \& Johnson, 1972; Craik \& Lockhart, 1972; Craik \& Tulving, 1975; Hyde \& Jenkins, 1969; James, 1899; Jenkins, 1974; Kaneda, Shigemune, \& Tsukiura, 2017; Roediger, Meade, Gallo, \& Olson, 2014; Rumelhart, 1980). Of particular relevance to CHREST's assumption that the learning of templates is automatic is the conclusion of Jenkins (1974), who reviewed then-current memory research and stated: "First, it is surely clear that memory is not an automatic consequence of being exposed to external events" (p. 16). This conclusion would be challenged if CHREST could satisfactorily account for all of the relevant data. Moreover, if memory for something as complex as chess positions can be accounted for without appeal to high-level conceptual processes, then perhaps the role of these processes in other domains has been overstated.

Contrary to the tenets of template theory, and consistent with the just-noted studies on memory, several researchers have argued that the expertise effect in memory for chess positions is due, in part, to experts having more sophisticated high-level knowledge of chess (Cooke et al., 1993; Frey \& Adesman, 1976; Goldin, 1978; Lane \& Robertson, 1979; Linhares, Freitas, Mendes, \& Silva, 2012; Lories, 1987; Saariluoma, 2001). The focus of this article is on whether high-level conceptual processes play an important role in memory for chess positions. As was noted by Cooke et al., a conclusion that high-level processes play an important role would not imply that pattern recognition is not also important.

It is important for readers who are not experienced chess players to have some appreciation of the kind and extent of knowledge that chess players possess. As long ago as 1893, Binet (republished as Binet, 1966) stated that "The art of chess is also a science, and more than a thousand volumes of analysis have been written about it" (p. 147). Chassy and Gobet (2011) estimated that 50,000 or more chess books have been published.

For the sake of those with little familiarity with chess, we exemplify the kind of chess knowledge that strong chess players possess with one of the simpler lessons from Euwe's (1953) classic introductory book Judgment and Planning in Chess. In this example, Euwe explains what it means for a bishop to be a "bad bishop" and shows how an understanding of this high-level concept provides a basis for understanding the position and guides the choice of moves.

Figure 1 shows a position from a game presented in Euwe (1953). As Euwe explained, bishops on the same color squares as their pawns are "bad bishops" because their mobility is reduced. In Fig. 1, Black's bishop is blocked by the pawns on c6, $\mathrm{d} 5$, and f5. Therefore, Black's goal is to move some of these pawns to dark squares so they do not obstruct the light-squared bishop. If it were Black's move, Black could move the pawn from $\mathrm{f5}$ to $\mathrm{f} 4$, improving the scope of the bishop. However, it is White's move, and White moves the pawn from $\mathrm{f} 2$ to $\mathrm{f} 4$, preventing this advance. If high-level processes were important for chess memory, then recognizing this position as an example of a knight versus a bad bishop and all that that entails would be expected to help an expert player grasp the totality of the position and remember it better. Note that we are not claiming that identifying the position as knight versus bad bishop directly clues the player as to which pieces are on which squares. Instead, we suggest that recognizing the position as being knight versus bad bishop helps the player organize the position into a coherent whole, and that doing so aids recall. We 


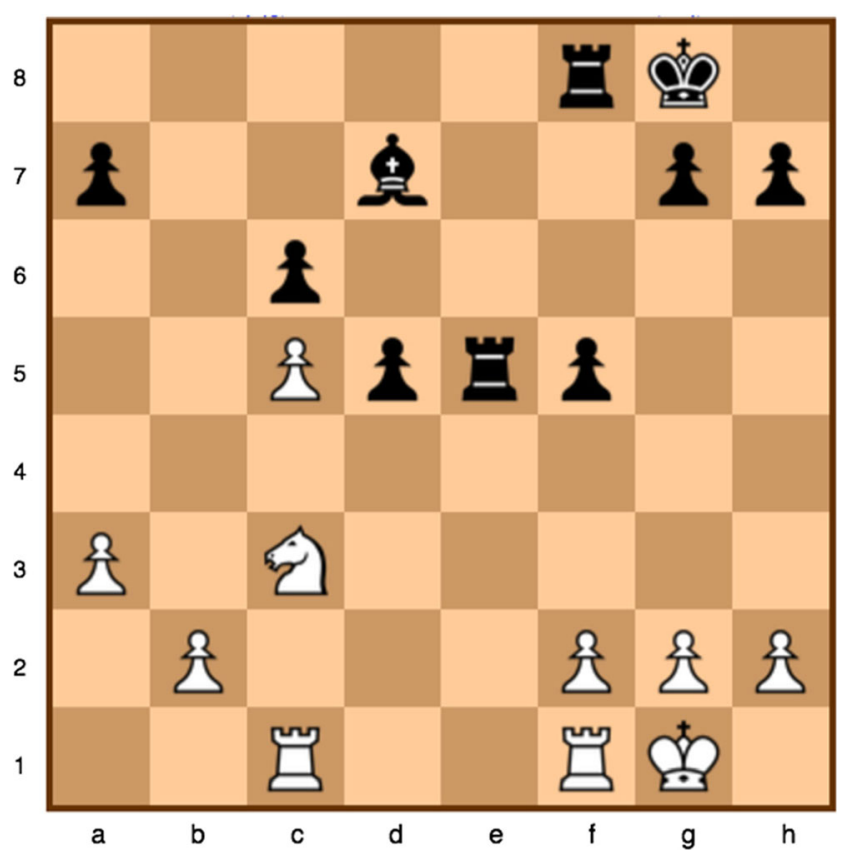

Fig. 1 This position exemplifies a knight versus a bad bishop. The image is a screen capture from chessgames.com, reprinted with permission.

recognize that there are other interpretations of the way that this position is memorized and recalled, and it is presented here simply as an example of how a high-level concept might be used in recall.

A review of research indicating a role for high-level concepts in memory for chess positions follows.

Interestingly, the importance of meaning for the recall of chess positions was recognized by Chase and Simon (1973), who stated:

There is one important mechanism, however, that is missing in the Simon-Gilmartin theory, as it is presently formulated. The simulation (unlike the SimonBarenfeld program) makes no provision for the perception of meaningful but unfamiliar patterns; only familiar patterns that are stored in long-term memory are recognized. (p. 249, emphasis added)

Lane and Robertson (1979) compared recall following semantic-orienting instructions ("find the best move and determine which side has the advantage"), formal-orienting instructions ("determine the number of pieces on light squares and the number of pieces on dark squares"), and intentionallearning instructions ("study the position so as to be able to recall it at a later point"). Supporting the role of high-level processes, they found that the semantic-orienting instructions and the intentional-learning instructions led to much higher levels of recall than did the formal-orienting instructions.

Baddeley and Hitch (2017) found that semantic-orienting instructions were superior to formal orienting instructions for both verbal and visual stimuli, and that the advantage was much greater for verbal stimuli. They argued that the relative ease of elaboration of verbal stimuli as compared to the visual stimuli was responsible for the larger effect with verbal stimuli. The large effect of encoding instructions found by Lane and Robertson (1979) suggests that semantic-encoding instructions for visually presented chess positions promote elaborated semantic encoding.

Gobet (1996) argued that the advantage of semanticencoding instructions found by Lane and Robertson (1979) may not be inconsistent with Chase and Simon's (1973) model, because "these experiments only invite players to encode different kinds of information into STM" (p. 109). It is not clear how inviting different kinds of encoding could be modeled by CHREST, since CHREST assumes automatic encoding and does not include a means to incorporate different kinds of encoding instructions. As was stated by Gobet and Lane (2005), "chunking mechanisms operate automatically and implicitly" (p. 211).

It might appear that these results could be accommodated by CHREST by assuming that the formal orienting instructions decreased the likelihood of recognizing a template. However, this explanation is hard to reconcile with CHREST's emphasis on automaticity and the suggestion by Gobet (1996) that automatisms increase with expertise and, at some level of expertise, the difference between formal and semantic orienting tasks would essentially vanish. Although the advantage of semantic- over formal-orienting instructions has not been tested with master-level players, Lane and Robertson's (1979) results cast doubt on the possibility that this advantage would essentially disappear. First, the advantage of semantic-orienting over formal-orienting instructions increased greatly as a function of expertise, making it unlikely that further increases in expertise would reduce this difference to near zero. Second, although the argument that performing a formal-orienting task makes it more difficult to recognize chunks and templates is plausible, one would not expect it to completely eliminate the expert advantage at recognizing templates. However, Lane and Robertson found absolutely no expertise effect in their formal-orienting condition.

Saariluoma and Kalakoski's (1998) results also argue against an interpretation of orienting-instruction effects based solely on the possibility that formal-orienting instructions interfere with pattern recognition. In their Experiment 2, Saariluoma and Kalakoski compared master chess players' recognition memory for chess positions after semanticorienting and formal-orienting instructions. In the learning phase, a series of positions were presented piece by piece, with only one piece appearing on the board at a time. One group of players was instructed to find the best move, whereas the other group was instructed to count the number of White pawns on White squares, Black pawns on Black squares, other White pieces on Black squares, and other Black pieces on White squares. In the test phase, positions that had not been 
presented previously consisted of positions that had been minimally transposed by changing the location of one piece. A critical manipulation was that the transposed piece could either be or not be functionally significant. Functional significance was defined a priori as pieces that chess commentators had identified as participating in the main analysis variation. Importantly, "functionally significant" was not equivalent to "geometrically central," and a functionally significant change could occur anywhere on the board.

If formal-orienting instructions interfere with recognizing patterns, then an analysis in terms of pattern recognition would suggest that performance would be better in the semanticorienting than in the formal-orienting condition. However, performance was essentially the same in these conditions. Critically, the interaction of orienting instructions and the functional significance of the transposed piece was very large and provided clear evidence of the importance of semantic processing. Specifically, for positions containing a functionally significant change, performance was better in the semantic-orienting than in the formal-orienting condition, whereas the opposite pattern was found for positions for which the change was not functionally significant. Moreover, the players in the semanticorienting condition performed extremely poorly on positions with nonfunctional changes, almost always identifying positions with these transpositions as having been presented before. This pattern of results is difficult to explain strictly in terms of pattern recognition, without regard to semantic processing.

Cooke, Atlas, Lane, and Berger (1993), based on the classic study of Bransford and Johnson (1972), provided subjects with high-level information about a chess position either before or after presenting the position. Positions were presented four pieces at a time, chosen randomly without replacement at intervals of $10 \mathrm{~s}$, until all the pieces had been presented. Recall was better in the "information-before" than in the "information-after" condition, supporting the thesis that the high-level information aided the encoding and eventual memory of the position. Gobet (1994) argued that template theory could explain this finding as follows:

The model also predicts that giving a high-level description before the presentation of a position enhances recall more than when it is given after (cf. Cooke et al., 1993): in the former case, the model (a) gains time to access the schema - it is provided to the player - and has then time to memorize smaller chunks or individual pieces, and (b) may use the schema to correct its default values or fill in slots without default value. (p. 36)

We believe it would be challenging for CHREST to model the results of Cooke et al. (1993). Before discussing these complexities, we will note an epistemological view adopted by Gobet and Simon (2000) that is relevant here. These authors claimed that a prediction made by Chase and Simon
(1973) regarding expertise effects for random positions was "incautious," because it was not based on a computational model and was therefore only a guess. The implications of this argument were summarized by Vicente (2000) as follows:

So, to generalize, when one has a formal process theory embedded in a computer simulation, a prediction made without running the simulation is incautious and should be treated only as a guess. (p. 606)

Although we believe that Gobet's explanation of Cooke et al.'s (1993) results is far more than a guess, it is important to note that there are considerable differences between the procedure used in the experiments modeled by CHREST and the procedure used by Cooke et al. The former consisted of showing a complete chess position for a short period of time, removing the position, and then asking the player to reconstruct it. The latter procedure consisted of presenting four pieces at a time and removing the pieces after a short time, until all the pieces within the position had been presented.

Consider the processes potentially involved during encoding following the presentation of a high-level description. Since templates are primarily specific configurations of pieces on squares, there is no way a player could know from the description which of the many templates consistent with the description should be used. When the first randomly chosen set of four pieces is shown, the player could attempt to narrow the set of possibly applicable templates and somehow keep them in mind. This process would have to be specified in detail to be modeled. A mechanism must also be specified for integrating the conceptual description of a position with the processing of pieces that, at the time they are presented, could be part of one of several candidate templates, could belong in slots for a template, or could be unrelated to a template. Without implementing the model, there is no way to be confident of the advantage, if any, that such model would predict for the information-before condition. This is not to say that modeling the results of Cooke et al. (1993) on the basis of template theory is necessarily impossible. For example, CHREST could be modified to associate templates with verbal descriptions. A verbal description provided before a position is presented could match many templates and chunks, and the model would select one that is consistent with information provided by the first eye fixation. If a better one is found later, it would replace the first one. However, the onus of proof that such a model is practical and accurate lies with the proponents of CHREST.

Comparing recall in the information-before condition with recall in the information-after condition controls for the informational effects of the description, and therefore controls for the player's ability to fill in a schema's default values or to fill in slots without default values. We do not find the argument that these factors allow the effect to be explained in terms of template theory completely convincing. 
Linhares, Freitas, Mendes, and Silva (2012) assessed whether chess positions are represented abstractly by analyzing the mistakes players made in reconstructions of positions. In an example indicating that positions are represented abstractly, a player reconstructed a position accurately at an abstract level by including a back-rank mate with the seventh rank controlled by a major piece. However, the major piece in the actual position was the queen, whereas the major piece playing the same role in the reconstruction was a rook. Significantly, $52 \%$ of the pieces were either misplaced or omitted, despite the abstract representation being correct.

Gobet and his colleagues have argued that the ability of CHREST to predict an expertise effect for random positions makes it superior to approaches that emphasize high-level processes (Gobet, 1998b; Sala \& Gobet, 2017). Gobet (1998b) even included a section titled "Random Positions as a Litmus Test for Theories of Expert Memory" (p. 400). Although CHREST does an impressive job accounting for the expertise effect in the recall of random positions in terms of pattern recognition, it should be kept in mind that pattern recognition and higher-level processing are not mutually exclusive. Moreover, Goldin (1979) provided evidence that meaningfulness plays a role even in random positions. In her study, she found that "all four of the high-skill subjects reported they looked for normal, analyzable subpatterns even in the scrambled stimuli and used these analyzed sections later as a basis for recognition" (p. 28).

The task of modeling behavior is clearly more complex than simply demonstrating that higher-level concepts play a role in chess memory. Although these demonstrations do not support specific theories of chess recall, they do provide basic data that must be explained by a complete theory of chess memory. We believe the studies we have just reviewed pose serious challenges to CHREST. However, research is typically subject to alternative interpretations, and therefore it is not unlikely that some researchers will disagree with us about the implications of these studies for the status of CHREST. Moreover, it is possible, in principle, that CHREST or another model based on pattern recognition could account for the findings that purport to show an important role for high-level processes. A good historical analogue can be found in the excellent article by Spence (1936), showing that behavior apparently demonstrating hypotheses in rats (Krechevsky, 1932) could be modeled solely on the basis of the gradual buildup and inhibition of the "excitatory potential" evoked by stimuli. ${ }^{1}$

Correlational studies are consistent with both template theory and the view that high-level processes play an important role in chess memory. Relevant to template theory is the

\footnotetext{
${ }^{1}$ Lawrence (1949) demonstrated that the distinctiveness of cues is learned and that a theory based only on excitatory potential was inadequate. Subsequently, Zeaman and House (1963) developed a two-process model in which subjects slowly learned which dimension to pay attention to (explaining Lawrence's data) and subsequently quickly learned the required response to the relevant cue (analogous to Spence's buildup of excitatory potential).
}

finding of a strong relationship between the number of hours a player has studied and/or played chess and that player's chess rating (Chang, 2016; Charness, Tuffiash, Krampe, Reingold, \& Vasyukova, 2005; Gobet \& Campitelli, 2007). It follows that chess experts will have had more opportunities to learn chunks and templates than have lesser players. According to template theory, this more extensive learning of and later recognition of chunks and templates is responsible for the expertise effect in chess recall. On the other hand, the number of hours a player has studied and/or played chess is almost certainly related to a player's understanding of chess positions. Since there is a strong correlation between a player's chess knowledge and chess rating (Pfau \& Murphy, 1988), a chess expert would be expected to have a better conceptual grasp of the game and would be better able to use these high-level concepts for remembering chess positions.

It is difficult to know how much of the effect on memory performance is due to pattern recognition and how much is due to high-level concepts, because experience increases both the learning of patterns and the learning of high-level concepts. However, knowledge of patterns and knowledge of high-level concepts are unlikely to be perfectly correlated, because pattern knowledge is an automatic function of exposure, whereas high-level knowledge is likely also due to other factors, including the manner in which chess is studied and cognitive ability. This means that the portion of high-level knowledge that is independent of the time spent on chessrelated activities can be used as a measure of high-level knowledge unconfounded with knowledge of patterns.

The magnitude of the correlation of this measure and memory for chess positions reflects the importance of high-level knowledge for memory for chess positions. A zero or negligible correlation would represent a lack of evidence for an important role for high-level concepts and, following Occam's razor, would indicate that an explanation strictly in terms of pattern recognition is preferable to one invoking high-level processing. On the other hand, a nonnegligible correlation would indicate that a model ignoring high-level processes is not fully adequate. Naturally, researchers may disagree about how high a correlation has to be before it is considered nonnegligible.

In the present study, we assessed the time players had spent on chess-related activities that are assumed to promote the learning of patterns and/or templates, their declarative knowledge of chess, their fluid intelligence, and their ability to remember chess positions. As we just discussed, the key question is whether declarative knowledge of chess is related to chess memory even after controlling for the time available for learning templates and/ or patterns. An advantage of using this correlational approach is that the independent contribution of high-level processes is assessed without regard to the details of any specific pattern recognition model of memory. As a result, if the data support the conclusion that high-level knowledge plays an important role 
in memory for chess positions, this conclusion could not be diminished by the assertion that a modified model based solely on pattern recognition could, in principle, account for the data.

We were also interested in the role of cognitive ability, because if cognitive ability plays an important role in chess memory, then, in order to provide full accounts, theories and models of chess memory would have to include individual differences in cognitive ability. There is strong evidence that cognitive ability plays a role in chess expertise (Burgoyne et al., 2016; Sala et al., 2017).

\section{Method}

\section{Subjects}

A total of 79 chess players were recruited from different chess clubs and chess tournaments in various Texas cities-Dallas, Fort Worth, College Station, Beaumont, Galveston, Houston, and surrounding areas - with one recruited in Taiwan. Two subjects were excluded from the analysis: One was excluded because he did not perform the chess knowledge task, and the second because he did not fill out the survey concerning the amounts of time studying alone and playing chess. The remaining sample of 77 chess players consisted of 67 males and 10 females, with a mean age of 35 years; their ages ranged from 18 to 77 years old. The chess players had a wide range of USCF chess ratings $(\min .=381$, max. $=2651$, mean $=1683$, median $=$ $1742, S D=574$, skew $=-.34$ ), with no values approaching an outlier based on Wilcox and Keselman's (2003) recommended outlier detection method (Formula 3, p. 264).

\section{Procedure}

Subjects were given a chess memory test, a chess knowledge test, a questionnaire asking about their chess experience, and a test of fluid intelligence. We determined the number of rated games that players had played from chess databases.

Chess knowledge test We used van der Maas and Wagenmakers's (2005) Verbal Knowledge Test, which had been adapted from the Amsterdam Chess Test, which itself was adapted from Pfau and Murphy's (1988) knowledge test. This test consists of 18 four-alternative multiple-choice questions varying in difficulty: Five questions refer to opening knowledge, four questions to strategic/positional knowledge, five questions to endgame knowledge, one question regarding a definition of chess, and three questions measuring the capacity to visualize chess moves. Because we were interested in conceptual knowledge and not in chess visualization ability, we did not include these visualization questions in the analyses presented here.

Critically, this modified version of the chess knowledge test contained no items that could be answered on the basis of knowledge of patterns of chess pieces. An example question follows, showing that the declarative knowledge required for the test had no relationship to patterns of pieces:

In which opening will White often castle queenside?
a. French
b. Sicilian
c. Ruy Lopez
d. Polish

Chess memory test We used the memory test from van der Maas and Wagenmakers (2005). This test is based on six realistic positions varying in the number of pieces in the position. Each position was presented for $10 \mathrm{~s}$, followed by a blank screen for $2 \mathrm{~s}$. Subjects then attempted to reconstruct the positions on a physical chess board. The percentage of accurately reconstructed pieces was calculated.

Chess experience survey The participants were asked to fill out a shortened version of a survey adapted from Charness et al. (2005). Players were asked to estimate (1) the amount of time they had spent seriously analyzing positions alone (e.g., using chess books, magazines, databases) and (2) the amount of time they had spent seriously playing chess with opponents outside of tournaments. Estimates were given for a typical week, and these estimates were multiplied by the relevant number of weeks.

Tournament games played The numbers of games played in FIDE and USCF tournaments were obtained from the official websites of these organizations: https://www.fide.com/ and www.uschess.org/.

Fluid intelligence The fluid intelligence measure was based on performance on seven cognitive tasks: digit-span forward, digit-span backward, approximate number system, blocktapping forward, block-tapping backward, auto symmetry span, and visual short-term memory. Chang (2016) did a principal components analysis on these tasks and found that one factor accounted for $53 \%$ of the variance whereas the second factor accounted for only $13 \%$ of the variance. All seven of the cognitive tests had high loadings on this factor with the loadings ranging from .66 to .81. See Chang (2016) for details.

Note that our original intention was to investigate the role of various cognitive abilities on chess cognition rather than fluid intelligence per se. However, the finding that a onefactor solution provided a good summary of the data led us to do subsequent analyses using the first factor rather than the individual measures. We felt that "fluid intelligence" would be an appropriate name for this factor because of the nature of the tests that define it. Therefore, the factor scores on the first factor were used as a measure of fluid intelligence. 


\section{Results}

\section{Analysis plan}

The analysis plan was to use hierarchical multiple regression to assess how much chess knowledge contributed to chess memory after controlling for chess experience. The key question was how much adding chess knowledge to a model that already included measures of chess experience would increase the model's $R^{2}$. This method is conservative because the variance common to experience and knowledge does not contribute to this increase in $R^{2}$. The effect of memory and the effect of fluid intelligence were assessed in terms of the increases in $R^{2}$ that occurred after adding these variables the model. Knowledge was added following the experience variables, and fluid intelligence was added last. The logic for adding fluid intelligence last was that the variance common to knowledge and fluid intelligence was assumed to be primarily due to fluid intelligence's effect on knowledge and knowledge's subsequent effect on memory. Thus, the variance attributed to knowledge would consist of both the direct effect of knowledge and the indirect effect of fluid intelligence acting through knowledge. Only fluid intelligence's direct effect would be attributed to fluid intelligence. Although unlikely, it is theoretically possible that the relationship between the parts of knowledge explained by fluid intelligence and memory is only spurious. Therefore, in addition to the analysis just described, we planned to calculate the variance due to knowledge, eliminating its variance in common with either experience or fluid intelligence.

Computationally, variance common between variables is attributed to the variable(s) entered earlier in the multiple regression model. Therefore, different ways of attributing the common variance were computed by changing the order in which the variables were entered into the equation.

The first planned step was to examine whether the distributions of the variables deviated greatly from normality. Although the normality of predictor variables in regression is not assumed, reducing skew typically increases the strengths of relationships and power. The second planned step was to assess whether there was a violation of the regression assumption that the residuals would be normal. Finally, we planned to assess the increases in $R^{2}$ due to including chess knowledge and then fluid intelligence.

\section{Basic statistics}

The number of nontournament games played, the number of tournament games played, and the time spent studying alone all had very skewed distributions. In the computation of inferential statistics, a $\log _{10}$ transformation was applied to the former two variables in order to reduce skew, and a $\log _{10}(X+1)$ transformation was applied to the last variable because one player reported spending no time studying alone. The distributions of chess knowledge and chess memory did not deviate sufficiently from normality to warrant transformations.

Descriptive statistics for the variables are shown in Table 1, and the correlations among them are shown in Table 2. As can be seen in Table 2, memory was highly correlated with chess knowledge $(r=.67, p<.01)$, number of tournament games $(r=.59, p<$ $.01)$, and fluid intelligence $(r=.57, p<.01)$; it was moderately correlated with time studying alone $(r=.31, p<.01)$.

\section{Multiple regression analysis}

A multiple regression analysis predicting memory from the five variables resulted in an $R^{2}$ of .67 and an adjusted $R^{2}$ of .65. The distribution of residuals was approximately normal, with only one observation deviating substantially from expectations under the assumption of normality. Specifically, the player whose memory was the most overpredicted by the model had a standardized residual of -3.04 when, under the assumption of normality, the expected standardized residual was -2.23 . Because the assumption of normality is never exactly met (Box, 1976), inferential tests of deviations from normality are not very informative. Nonetheless, it is worth noting that the Shapiro-Wilk $W$ test indicated that the degree the residuals deviated from normality was less than would have be expected by chance, given that the population distribution was normal.

Estimating the variance explained by each variable was complicated by the fact that the variables were correlated, and as a consequence, much of the variance was common and the effects were confounded. To test the role of knowledge conservatively, we apportioned all the variance common between the experience variables (nontournament games, tournament games, and studying alone) and the other variables (knowledge and fluid intelligence) to the experience variables. Apportioning the common variance in this way, $38 \%$ of the

Table 1 Descriptive statistics

\begin{tabular}{lllllll}
\hline & Memory & Non-Tournament Games (h) & Studying Alone (h) & Tournament Games & Chess Knowledge & Fluid Intelligence \\
\hline Mean & 0.62 & 1,701 & 4,741 & 397 & .52 & 0.00 \\
Median & 0.63 & 428 & 2,080 & 107 & .53 & .20 \\
SD & 0.23 & 2,490 & 6,326 & 587 & .13 & 1.92 \\
Min. & 0.03 & 4 & 0 & 1 & .87 & -4.15 \\
Max. & 1.00 & 10,756 & 31,720 & 2,690 & 3.69 \\
\hline
\end{tabular}


Table 2 Pearson correlations

\begin{tabular}{|c|c|c|c|c|c|c|}
\hline & Memory & Non-Tournament Games (h) & Studying Alone $(\mathrm{h})$ & Tournament Games & Chess Knowledge & Fluid Intelligence \\
\hline Memory & 1.00 & .09 & .31 & .59 & .67 & .57 \\
\hline Nontourn. & .09 & 1.00 & .49 & .35 & .11 & -.05 \\
\hline Studying & .31 & .49 & 1.00 & .43 & .29 & .31 \\
\hline Tourn. & .59 & .35 & .43 & 1.00 & .53 & .21 \\
\hline Chess Know. & .67 & .11 & .29 & .53 & 1.00 & .37 \\
\hline Fluid & .57 & -.05 & .31 & .21 & .37 & 1.00 \\
\hline
\end{tabular}

Nontournament games, study alone, and tournament games were log-transformed for this analysis. All correlations of .29 and above have $p$ values $\leq .01$. No other correlations have $p$ values $<.05$.

variance was explained by the combination of the three experience variables, and $29 \%$ more was explained by the combination of knowledge and fluid intelligence. We attributed the variance common between knowledge and fluid intelligence to knowledge, because any effect of fluid intelligence on memory mediated through knowledge was, for the present purposes, best interpreted as a knowledge effect, since fluid intelligence can affect chess knowledge but chess knowledge cannot plausibly affect fluid intelligence. With this rule for apportioning common variance, $16 \%$ of the variance in memory was attributable to knowledge, and $13 \%$ to fluid intelligence. If the common variance were attributed to fluid intelligence, $7 \%$ of the variance would be attributable to knowledge and $22 \%$ to fluid intelligence. Similarly, the percentage of variance attributed to the experience variables would drop from $38 \%$ to $28 \%$, and the variance attributed to fluid intelligence would increase to $33 \%$ if the variance common to fluid intelligence and other variables were attributed to fluid intelligence alone. Finally, the percentage of variance attributed to the experience variables would drop to $10 \%$ if no common variance were attributed to the experience variables. All percentages of variance reported here are significantly greater than $0, p<$ .01 . More details on these regression analyses are presented in the Appendix.

Figure 2 illustrates the relationship between knowledge and memory with a partial regression plot (Velleman \& Welsch, 1981). Partial regression plots display the relationship between one variable in the model and the criterion, with other variables controlled. Figure 2 is based on a model omitting

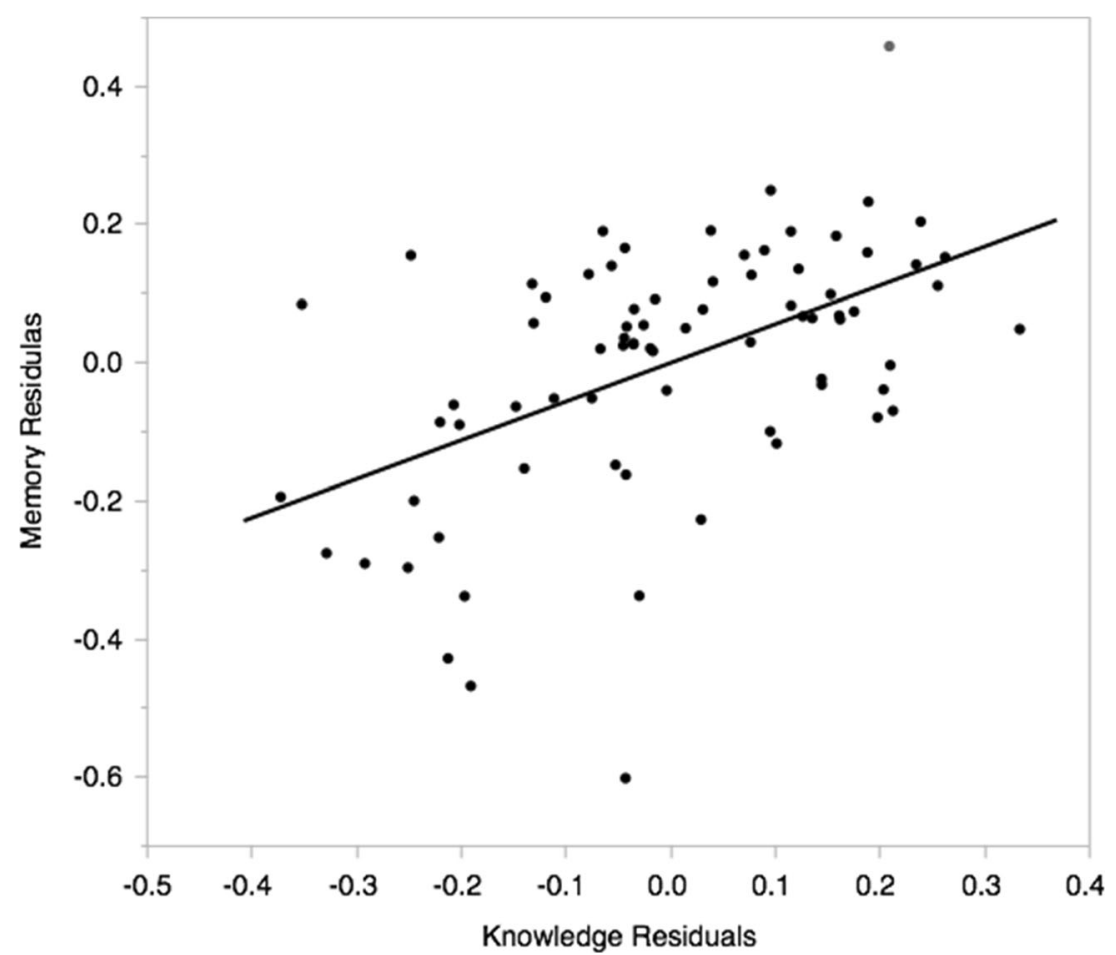

Fig. 2 Partial regression plot showing the part of memory independent of the three experience variables, as a function of the part of knowledge independent of these same variables. 
fluid intelligence, so as to be consistent with the way we attributed the variance confounded between knowledge and fluid intelligence to knowledge. The substantial relationship between knowledge and memory after controlling for the experience variables is evident.

\section{Discussion}

The findings that, even after controlling for chess experience, chess knowledge explains $16 \%$ of the variance in chess memory and that fluid intelligence explains an additional $13 \%$ means that a full account of memory for chess positions cannot be based entirely on pattern recognition: The more players know about chess, the higher their level of recall. Both recognition of patterns and high-level conceptual processing appear to play important roles in memory for chess positions.

CHREST, a sophisticated model of chess memory based on the learning and recalling of familiar patterns, has been successful modeling many phenomena. In principle, CHREST could be modified to incorporate high-level processes. However, the difficulty of doing this should not be underestimated. One particular challenge is to model how experts understand a position. Consider the master in Chase and Simon's (1973) study whose recall of one position was relatively poor. The master reported that he could not "get the sense" of the position. Clearly a model based on the recognition of patterns would have to be supplemented in a very sophisticated way to predict both the low level of recall and the self-report.

Consistent with the comment of Chase and Simon's (1973) master, Wolff, in describing how he memorized chess positions, stated that "The important thing is to try and make everything make sense" (Chabris \& Simons, 1998). The following description gives some detail about what Wolff meant when he said he tried to make sense of a position.

What I was trying to do was to just sort of absorb the position and understand where everything was clustered and try to quickly understand what was going on. Establish some logical connections between things ...

Tellingly, Wolff explained why he was unable to remember the position of a rook in one position. In the position, White had a winning move, and that move dominated his perception of the position. He described aspects of the position unrelated to this move as being hard to pay attention to and difficult.

This is a little bit tricky actually because White is going to win by playing bishop a7 and that sort of dominates everything and so that's why I can't remember where this rook is very well because the most important thing is this [pointing] and this [pointing] and this pawn structure [pointing] and this [pointing] is happening and it's just sort of hard to pay attention to the rest.

The difficulty of modeling Wolff's failure to recall the position of the rook is considerable, because the model would have to recognize the importance of the move Bishop to a7 and model the effect of the importance of this move on the perception of all other aspects of the position.

It is likely that higher fluid intelligence increases the ability to benefit from high-level processing more than it increases the ability to benefit from the automatic recognition of familiar patterns. Therefore, the finding that fluid intelligence is strongly related to chess memory further supports the argument that chess memory cannot be explained solely in term of recognizing patterns and that high-level processes play at least some role.

The high correlation of fluid intelligence with memory adds to the complexity of developing a complete computational model since this finding implies that mean performance may not fully represent any given individual's performance, a phenomenon that has been discussed frequently (Chuderski, Stettner, \& Orzechowski, 2007; Estes, 1956; Estes \& Maddox, 2005; Navarro, Griffiths, Steyvers, \& Lee, 2006; Rouder, Lu, Morey, Sun, \& Speckman, 2008). For the most part, CHREST has modeled mean performance, although its analysis of Gobet's own performance is a notable exception (Gobet \& Ritter, 2000).

As we noted in the introduction to this article, we believe that many findings have not yet been satisfactorily modeled by CHREST: a master reporting that his poor recall was because he could not get a sense of the position (Chase \& Simon, 1973); the effect of encoding instructions (Lane \& Robertson, 1979; Saariluoma \& Kalakoski, 1998); the effect of presenting conceptual information prior to the presentation of the chess position (Cooke et al., 1993); a player's getting the abstract relations in a position correct while making many errors placing pieces on squares (Linhares et al., 2012); and the failure of a player to recall the position of a particular piece because a winning move dominated the player's attention (Chabris \& Simons, 1998). As was emphasized by Simon and Gobet (2000), stating that a model can explain a phenomenon and actually modeling it are very different things. A common theme among these findings is that they demonstrate that high-level processes play an important role in memory for chess positions. The current finding of a strong relationship between chess knowledge and chess memory even after controlling for chess experience is further evidence that high-level processes are at least partially responsible for the expertise effect in chess memory. 
High-level conceptual processing and the low-level recognition of familiar patterns are not incompatible (Charness, 2012; Cooke et al., 1993; Gobet \& Simon, 1996), and there is strong evidence that both play important roles in memory for chess positions. We would emphasize that no current theory of chess memory is based entirely on high-level knowledge, and therefore the conclusion of this article is not that such a theory is or would be better than CHREST. Instead, our conclusion is that high-level processes play a sufficiently important role that they must to be taken into account in any complete theory. Finally, the conclusion that high-level processes are important in memory for chess positions is consis- tent with data and theories from other areas of memory research cited in the introduction.

Author note Partial funding was provided by the Social Science Research Institute at Rice University. The data for this article were collected as part of the second author's dissertation (Chang, 2016), which was designed to investigate issues related to individual differences in chess skill rather than the basis of the expertise effect in memory for chess positions. As it turns out, her data are relevant to the latter problem as well as to the former.

\section{Appendix}

\begin{tabular}{|c|c|c|c|c|c|}
\hline \multicolumn{6}{|c|}{ Regression of Memory on Experience } \\
\hline Term & $b$ & Standard Error & $t$ & $p$ & beta \\
\hline $\log ($ Study Alone +1$)$ & .051 & .037 & 1.370 & .175 & .152 \\
\hline Log(Playing) & -.068 & .039 & -1.75 & .084 & -.188 \\
\hline Log(Playing Tournaments) & .091 & .026 & 3.46 & .001 & .303 \\
\hline
\end{tabular}

$R^{2}=.382$

\begin{tabular}{|c|c|c|c|c|c|}
\hline Term & $b$ & Standard Error & $t$ & $p$ & beta \\
\hline Fluid intelligence & .045 & .010 & 4.59 & $<.001$ & .374 \\
\hline Chess knowledge & .628 & .096 & 6.57 & $<.001$ & .536 \\
\hline
\end{tabular}

$R^{2}=.574$

\begin{tabular}{|c|c|c|c|c|c|}
\hline Term & $b$ & Standard Error & $t$ & $p$ & beta \\
\hline Log(Study Alone + 1) & .028 & .033 & 1.49 & .140 & .085 \\
\hline Log(Playing) & -.044 & .034 & -1.28 & .204 & -.120 \\
\hline Log(Playing Tournaments) & .104 & .031 & 3.40 & .001 & .347 \\
\hline Chess knowledge & .561 & .111 & 5.04 & $<.001$ & .479 \\
\hline
\end{tabular}

$R^{2}=.543$

\begin{tabular}{|c|c|c|c|c|c|}
\hline \multicolumn{6}{|c|}{ Regression of Memory on Experience, Knowledge, and Fluid Intelligence } \\
\hline Term & $b$ & Standard Error & $t$ & $p$ & beta \\
\hline $\log ($ Study Alone +1$)$ & .058 & .028 & 2.05 & .045 & .173 \\
\hline Log(Playing) & -.041 & .029 & -1.42 & .161 & -.113 \\
\hline Log(Playing Tournaments) & .091 & .026 & 3.46 & .001 & .303 \\
\hline Chess knowledge & .387 & .101 & 3.84 & $<.001$ & .330 \\
\hline Fluid intelligence & .047 & .009 & 5.26 & $<.001$ & .395 \\
\hline
\end{tabular}

$R^{2}=.671$ 


\section{References}

Baddeley, A. D., \& Hitch, G. J. (2017). Is the levels of processing effect language-limited? Journal of Memory and Language, 92, 1-13.

Bartlett, F. C. (1932). Remembering: A study in experimental and social psychology. Cambridge, UK: Cambridge University Press.

Binet, A. (1966). Mnemonic virtuosity: A study of chess players [Trans. M. L. Simmel \& S. B. Barron]. Genetic Psychology Monographs, $74,127-162$

Box, G. E. (1976). Science and statistics. Journal of the American Statistical Association, 71, 791-799.

Bransford, J. D., \& Johnson, M. K. (1972). Contextual prerequisites for understanding: Some investigations of comprehension and recall. Journal of Verbal Learning and Verbal Behavior, 11, 717-726.

Burgoyne, A. P., Sala, G., Gobet, F., Macnamara, B. N., Campitelli, G., \& Hambrick, D. Z. (2016). The relationship between cognitive ability and chess skill: A comprehensive meta-analysis. Intelligence, 59, 72-83. https://doi.org/10.1016/j.intell.2016.08.002

Chabris, C, \& Simons, D. (1998). Chess expertise: Extraordinary memory for chess positions. Accessed from https://youtu.be/ rWuJqCwfjjc on 3/2/17.

Chang, Y. (2016). It takes more than practice and experience to become a chess master: Evidence from a child prodigy and from adult tournament players (Doctoral Dissertation). Rice University, Houston, TX.

Charness, N. (1976). Memory for chess positions: Resistance to interference. Journal of Experimental Psychology: Human Learning and Memory, 2, 641-653.

Charness, N. (1981). Aging and skilled problem solving. Journal of Experimental Psychology: General, 110, 21-38.

Charness, N. (2012). Patterns of theorizing about chess skillCommentary on Linhares and Freitas (2010) and Lane and Gobet (2011). New Ideas in Psychology, 30, 322-324.

Charness, N., Tuffiash, M., Krampe, R., Reingold, E., \& Vasyukova, E. (2005). The role of deliberate practice in chess expertise. Applied Cognitive Psychology, 19, 151-165.

Chase, W. G., \& Simon, H. A. (1973). The mind's eye in chess. In W. G. Chase (Ed.), Visual information processing (pp. 215-281). New York, NY: Academic Press.

Chassy, P., \& Gobet, F. (2011). Measuring chess experts' single-use sequence knowledge: An archival study of departure from "theoretical" openings. PLOS ONE, 6, e26692. https://doi.org/10. 1371/journal.pone.0026692

Chuderski, A., Stettner, Z., \& Orzechowski, J. (2007). Computational modeling of individual differences in short term memory search. Cognitive Systems Research, 8, 161-173.

Cooke, N. J., Atlas, R. S., Lane, D. M., \& Berger, R. C. (1993). Role of high-level knowledge in memory for chess positions. American Journal of Psychology, 106, 321-351.

Craik, F. I. M., \& Lockhart, R. S. (1972). Levels of processing: A framework for memory research. Journal of Verbal Learning and Verbal Behavior, 11, 671-684. https://doi.org/10.1016/S0022-5371(72) 80001-X

Craik, F. I. M., \& Tulving, E. (1975). Depth of processing and the retention of words in episodic memory. Journal of Experimental Psychology: General, 104, 268-294. https://doi.org/10.1037/00963445.104.3.268

de Groot, A. D. (1965). Thought and choice in chess. The Hague, The Netherlands: Mouton.

Estes, W. K. (1956). The problem of inference from curves based on group data. Psychological Bulletin, 53, 134-140.

Estes, W. K., \& Maddox, W. T. (2005). Risks of drawing inferences about cognitive processes from model fits to individual versus average performance. Psychonomic Bulletin \& Review, 12, 403-408. https://doi.org/10.3758/BF03193784
Euwe, M. (1953). Judgment and planning in chess. New York, NY: David McKay.

Feigenbaum, E. A. (1961). The simulation of verbal learning behavior. In Proceedings of the 1961 Western Joint Computer Conference (Vol. 19, pp. 121-132). New York, NY: ACM.

Feigenbaum, E. A., \& Simon, H. A. (1984). EPAM-like models of recognition and learning. Cognitive Science, 8, 305-336.

Frey, P. W., \& Adesman, P. (1976). Recall memory for visually presented chess positions. Memory \& Cognition, 4, 541-547.

Gobet, F. (1994). Memory in chess players: Chunks, schemata, or both? (Complex Information Processing Working Paper No. 517). Pittsburgh, PA: Department of Psychology, Carnegie Mellon University.

Gobet, F. (1996). Chess players' memory and perception: Recent work. In A. D. de Groot, F. Gobet, \& R. W. Jongman (Eds.), Perception and memory in chess: Studies in the heuristics of the professional eye (pp. 97-119). Assen, The Netherlands: Van Gorcum.

Gobet, F. (1998a). Expert memory: A comparison of four theories. Cognition, 66, 115-152.

Gobet, F. (1998b). Memory for the meaningless: How chunks help. In M. A. Gernsbacher \& S. J. Derry (Eds.), Proceedings of the Twentieth Annual Conference of the Cognitive Science Society (pp. 398-403). Mahwah, NJ: Erlbaum.

Gobet, F. (2013). Chunks and templates in semantic long-term memory: The importance of specialization. In J. J. Staszewski (Ed.), Expertise and skill acquisition: The impact of William G. Chase (pp. 117146). New York, NY: Psychology Press.

Gobet, F. (2016). Understanding expertise: A multi-disciplinary approach. New York, NY: Palgrave Macmillan.

Gobet F., \& Campitelli, G. (2007). The role of domain-specific practice, handedness and starting age in chess. Developmental Psychology, $43,159-172$

Gobet, F., \& Clarkson, G. (2004). Chunks in expert memory: Evidence for the magical number four . . . or is it two? Memory, 12, 732-747.

Gobet, F., \& Lane, P. C. (2005). The CHREST architecture of cognition: Listening to empirical data. In D. N. Davis (Ed.), Visions of mind: Architectures for cognition and affect (pp. 204-224). Hershey, PA: Information Science Publishing.

Gobet, F., Lane, P. C., Croker, S., Cheng, P. C., Jones, G., Oliver, I., \& Pine, J. M. (2001). Chunking mechanisms in human learning. Trends in Cognitive Sciences, 5, 236-243.

Gobet, F., Lane, P. C., \& Lloyd-Kelly, M. (2015). Chunks, schemata, and retrieval structures: Past and current computational models. Frontiers in Psychology, 6, 1785. https://doi.org/10.3389/fpsyg. 2015.01785

Gobet, F., \& Ritter, F. E. (2000). Individual data analysis and Unified Theories of Cognition: A methodological proposal. In N. Taatgen \& J. Aasman (Eds.), Proceedings of the 3rd International Conference on Cognitive Modeling (pp. 150-157). Veenendaal, The Netherlands: Universal Press.

Gobet, F., \& Simon, H. A. (1996). Templates in chess memory: A mechanism for recalling several boards. Cognitive Psychology, 31, 1-40.

Gobet, F., \& Simon, H. A. (2000). Five seconds or sixty? Presentation time in expert memory. Cognitive Science, 24, 651-682.

Goldin, S. E. (1978). Effects of orienting tasks on recognition of chess positions. American Journal of Psychology, 91, 659-671.

Goldin, S. E. (1979). Recognition memory for chess positions: Some preliminary research. American Journal of Psychology, 92, 19-31. https://doi.org/10.2307/1421476

Gong, Y., Ericsson, K. A., \& Moxley, J. H. (2015). Recall of briefly presented chess positions and its relation to chess skill. PLoS ONE, 10, e0118756. https://doi.org/10.1371/journal.pone.0118756

Hyde, T. S., \& Jenkins, J. J. (1969). Differential effects of incidental tasks on the organization of recall of a list of highly associated words. Journal of Experimental Psychology, 82, 472-481. 
James, W. (1899). Talks to teachers about psychology. New York, NY: Henry Holt \& Co.

Jenkins, J. J. (1974). Can we have a theory of meaningful memory? In R. L. Solso (Ed.), Theories in cognitive psychology: The Loyola Symposium (pp. 1-20). Potomac, MD: Erlbaum

Kaneda, T., Shigemune, Y., \& Tsukiura, T. (2017). Lateral and medial prefrontal contributions to emotion generation by semantic elaboration during episodic encoding. Cognitive, Affective, \& Behavioral Neuroscience, 17, 143-157.

Krechevsky, I. (1932). "Hypotheses" in rats. Psychological Review, 39, 516-532. https://doi.org/10.1037/h0073500

Lane, D. M., \& Robertson, L. (1979). The generality of the levels of processing hypothesis: An application to memory for chess positions. Memory \& Cognition, 7, 253-256.

Lawrence, D. H. (1949). Acquired distinctiveness of cues: I. Transfer between discriminations on the basis of familiarity with the stimulus. Journal of Experimental Psychology, 39, 770-784.

Linhares, A., Freitas, A. E. T., Mendes, A., \& Silva, J. S. (2012). Entanglement of perception and reasoning in the combinatorial game of chess: Differential errors of strategic reconstruction. Cognitive Systems Research, 13, 72-86.

Lories, G. (1987). Recall of random and non random chess positions in strong and weak chess players. Psychologica Belgica, 27, 153-159.

Navarro, D. J., Griffiths, T. L., Steyvers, M., \& Lee, M. D. (2006). Modeling individual differences using Dirichlet processes. Journal of Mathematical Psychology, 50, 101-122. https://doi.org/10.1016/ j.jmp.2005.11.006

Pfau, H. D., \& Murphy, M. D. (1988). Role of verbal knowledge in chess skill. American Journal of Psychology, 101, 73-86.

Richman, H. B., Staszewski, J. J., \& Simon, H. A. (1995). Simulation of expert memory using EPAM IV. Psychological Review, 102, 305330. https://doi.org/10.1037/0033-295X.102.2.305

Roediger, H. L., Meade, M. L., Gallo, D. A., \& Olson, K. R. (2014). Bartlett revisited: Direct comparison of repeated reproduction and serial reproduction techniques. Journal of Applied Research in Memory and Cognition, 3, 266-271.

Rouder, J. N., Lu, J., Morey, R. D., Sun, D., \& Speckman, P. L. (2008). A hierarchical process-dissociation model. Journal of Experimental Psychology: General, 137, 370-389. https://doi.org/10.1037/00963445.137.2.370
Rumelhart, D. E. (1980). Schemata: The building blocks of cognition. In. R. Spiro, B. Bruce, \& W. Brewer (Eds.), Theoretical issues in reading comprehension (pp. 33-58). Mahwah, NJ: Erlbaum.

Saariluoma, P. (2001). Chess and content-oriented psychology of thinking. Psicológica, 22, 143-164.

Saariluoma, P., \& Kalakoski, V. (1998). Apperception and imagery in blindfold chess. Memory, 6, 67-90.

Sala, G., Burgoyne, A. P., Macnamara, B. N., Hambrick, D. Z., Campitelli, G., \& Gobet, F. (2017). Checking the "Academic Selection" argument: Chess players outperform non-chess players in cognitive skills related to intelligence. A meta-analysis. Intelligence, 61, 130-139.

Sala, G., \& Gobet, F. (2017). Experts' memory superiority for domainspecific random material generalizes across fields of expertise: A meta-analysis. Memory \& Cognition, 45, 183-193. https://doi.org/ 10.3758/s13421-016-0663-2

Schultetus, R. S., \& Charness, N. (1999). Recall or evaluation of chess positions revisited: The relationship between memory and evaluation in chess skill. American Journal of Psychology, 112, 555-569.

Simon, H. A., \& Gilmartin, K. (1973). A simulation of memory for chess positions. Cognitive Psychology, 5, 29-46.

Simon, H. A., \& Gobet, F. (2000). Expertise effects in memory recall: A reply to Vicente and Wang. Psychological Review, 107, 593-600. https://doi.org/10.1037/0033-295X.107.3.593

Spence, K. W. (1936). The nature of discrimination learning in animals. Psychological Review, 43, 427-449.

van der Maas, H. L. J., \& Wagenmakers, E.-J. (2005). A psychometric analysis of chess expertise. American Journal of Psychology, 118, 29-60.

Velleman, P. F., \& Welsch, R. E. (1981). Efficient computing of regression diagnostics. American Statistician, 35, 234-242.

Vicente, K. J. (2000). Revisiting the constraint attunement hypothesis: Reply to Ericsson, Patel, and Kintsch (2000) and Simon and Gobet (2000). Psychological Review, 107, 601-608. https://doi. org/10.1037/0033-295X.107.3.601

Wilcox, R. R., \& Keselman, H. J (2003). Modern robust data analysis methods: Measures of central tendency. Psychological Methods, 8 , 254-274. https://doi.org/10.1037/1082-989X.8.3.254

Zeaman, D., \& House, B. J. (1963). The role of attention in retardate discrimination learning. In N. R. Ellis (Ed.), Handbook of mental deficiency: Psychological theory and research (pp. 159-223). New York, NY: McGraw-Hill. 